\title{
Whale-crow optimization (WCO)-based Optimal Regression model for Software Cost Estimation
}

\author{
SUMERA W AHMAD* and G R BAMNOTE \\ Department of Computer Science and Engineering, Prof. Ram Meghe Institute of Technology and Research, \\ Badnera, Amravati 444 701, India \\ e-mail: sumeraahmad2003@gmail.com; grbamnote@mitra.ac.in
}

MS received 2 August 2018; revised 19 November 2018; accepted 21 December 2018; published online 27 March 2019

\begin{abstract}
Software Cost Estimation (SCE) is the emerging concern of the software companies during the development phase of the software, as it requires effort and cost factors for modelling the software. These factors are modelled using the Artificial Intelligence models, which seem to be less accurate and non-reliable by increasing the risk factor of the software projects. Thus, for estimating the software cost, meta-heuristics are employed. This paper proposes an algorithm, termed as whale-crow optimization (WCO) algorithm, which is the integration of the whale optimization algorithm (WOA) and the crow search algorithm (CSA). The main function of the WCO algorithm is to determine the Optimal Regression coefficients for the regression models, such as the Linear Regression model and the Kernel Logistic Regression model, to develop an Optimal Regression model to estimate the software cost. The experimentation is carried out using four datasets taken from the Promise software engineering repository to perform effective performance analysis. Analysis is carried out regarding the mean magnitude of relative error (MMRE) that proves that the proposed method of SCE is effective, attaining the average MMRE at a rate of 0.2442 for the proposed Linear Regression model and 0.2692 for the proposed Kernel Regression model.
\end{abstract}

Keywords. Kernel Logistics Regression model; Linear Regression model; WOA; CSA; Software Cost Estimation.

\section{Introduction}

The cost estimation is the main aspect in the software management projects, which contributes a lot to the project managers in analysing the feasibility of the project and thereby improves the effectiveness of the software development process [1]. Software Cost Estimation (SCE) creates and applies a required model for estimating the resources needed for a fully functional software system [2]. The software cost arises from developer time, training, managing and buying extra equipment, servers and software. SCE focuses on associating estimates of time and effort with the project activities. The cost of software is estimated by the available price that the customer has to spend on the project. The SCE improves the resource management by finding the resource requirements for every project and increases the resource efficiency by properly scheduling the resources. The cost models and the estimation techniques of software engineering are employed for a number of applications, such as project planning and authority, investment analysis for software improvement and so on [3]. Thus, the target over time is the need for

*For correspondence effective cost estimation methods [1, 4]. The SCE techniques are sub-divided into algorithmic and non-algorithmic. The function of the algorithmic method is to model the mathematical equations for performing the software estimation, which depends on the research and the historical data. There are various algorithmic models, like the COCOMO models [5], Putnam model [6] and models based on function points $[3,7]$.

The requirement of accurate cost estimation causes the exploration of the non-algorithmic models that depend on the machine learning algorithm as they make minimal assumptions regarding the function of the development under the study [8]. The cost estimation models rely on the historical data and develop the systems that learn from the data [9, 10]. The algorithmic models are based on the estimations that are done in the previous projects and previous experiences that are similar to the estimated project $[11,12]$. The algorithmic models use artificial intelligence (AI) methodologies, such as the genetic algorithm (GA), neural networks, rule-based induction and so on [13]. The non-algorithmic models are categorized as models based on neural network, fuzzy logic and GA. Neural networks are the nets of the processing elements that can learn between the inputs and the outputs $[14,15]$. The neurons obtain the 
output using the sum of the product of the weights and the inputs, and they generate the output if the sum is greater than the threshold. The output thus obtained becomes the positive or negative input to the neurons present in the network. The process is continued until the generation of the outputs [3, 16].

Fuzzy logic is a powerful tool that possesses the capacity to solve highly complex problems, or, in other words, the fuzzy logic tends to manage the mathematical model that is very tough or impossible to design [17]. There are parameters with certain levels of the fuzziness that are essential for the development of software [18]. Employing fuzzy logic has the property to tackle a few problems that exist in the existing estimation techniques [19]. The functions of fuzzy logic not only rely on the effort prediction but also on enhancing the quality of current estimating models. Moreover, Genetic Programming (GA) is an evolutionary algorithm used for the effort estimation, and the evolutionary computation is based on the fact that the solution is attained using the cycle of iterations [20, 21]. Researchers contribute various models [22] for estimation, yet a new method is required to depict the best estimate for all the situations as each technique is suitable only for a special project [23]. No one can say whether a method is better or worse than another, but they are complementary to each other. Thus, the sense of judgment proves that no other methods are better among themselves in the estimation software development effort [3]. The utilization of metaheuristic algorithms [24-27] plays an important role in SCE. These algorithms are used for accurate cost estimates for controlling software project.

The paper proposes an algorithm to determine the Optimal Regression coefficients of the SCE methods. The SCE is performed using two methods, namely the Linear Regression model and the Kernel Logistics Regression model. The Linear Regression model presents analysis of the linear relationship between software cost and the estimation cost attributes. The Linear Regression model is the summation of the product of the software attributes and the regression coefficients, whereas the Kernel Logistics Regression model is the summation of the product of the regression coefficients with the kernel function. A greedy algorithm is employed for determining the kernel function. The regression coefficients are optimally tuned using the proposed whale-crow optimization (WCO) algorithm, which determines the regression coefficients optimally so that the cost of the software is estimated efficiently.

The main contribution of the paper is the WCO algorithm, which aims at the optimal tuning of the regression coefficients of the SCE techniques, such as the Linear Regression model and the Kernel Logistics Regression model.

The organization of the paper is as follows. Section 1 presents the background of the paper, and the initiative of the paper that motivated the contribution of the new algorithm is depicted in section 2 . The proposed method used for the optimal estimation of the cost is deliberated in section 3, section 4 discusses the results of the proposed method and finally, section 5 provides the conclusion to the paper.

\section{Motivation}

\subsection{Related works}

In [13], SCE method using the ant colony optimization (ACO) and hybrid genetic algorithm was introduced that brought a proper balance among the estimation factors so that the estimation of the cost of the software was efficient. This method had enabled upgrading the software, controlling the software project and had ensured the quality of the software. This method offers lower estimation errors, but in case of a small number of population, convergence and achieving the near global optimum solution are major drawbacks. An SCE approach using the dissimilarity measure was introduced in [16]. The algorithm could be efficiently used for building models of SCE, but the output of this algorithm can be interpreted in several ways. An SCE approach using the Semi-Parametric models (SPMs) [2] checks whether the accuracy of the method was improved upon merging the non-parametric techniques with the linear component. Even though the SPMs provided very encouraging results, the method did not offer optimized results. A hybrid methodology to model the Morphological-RankLinear (MRL) perceptions in the issue of Software Developing Cost Estimation (SDCE) was proposed in [11]. Additionally, a modified genetic algorithm (MGA) was employed, which optimized the parameters of the MRL perception and selected the optimal input feature subset of the used databases such that higher accuracy was attained. Moreover, the gradient steepest descent method was applied for each of the MGAs in order to enhance the MRL perception parameters provided by the MGA. This method possesses less computational complexity, but the properties of this model need to be formalized. Araújo et al [12] introduce an evolutionary morphological approach, for which the hybrid artificial neuron is employed based on the Mathematical Morphology (MM). It possesses a number of advantages, like simple nonlinear components, and offers less computational complexity, but this method does not solve the regression problems. Sadiq and Shahid [14] analysed the software cost and risk using esrcTool that paved the way for the estimation of the cost of the software and paved the way for the design of the software, but the esrcTool was not applicable to real-world problems. An SCE using the Functional Link Artificial Neural Network (FLANN) and the Intuitionistic Fuzzy C-Means Clustering (IFCM) was introduced in [1]. The proposed FLANN-IFCM was found to be more effective in estimating the software, but the membership functions were not defined. Mittas and Angelis [15] propose a cost-effective model using a multiple comparisons 
algorithm, which is suitable for large-scale set-ups but suffers from inaccuracy issues.

Dizaji and Gharehchopogh [28] utilized the metaheuristic algorithm for SCE. Here, ACO and Lorentz transformation are utilized as chaos optimization algorithm (COA), and the performance of this algorithm is evaluated using the NASA datasets. The integration of the ACO algorithm with COA the improved performance. Gharehchopogh et al [29] introduced SCE by integrating the artificial bee colony (ABC) and GA. This hybrid algorithm has less MRE errors values than the COCOMO model. In addition, this algorithm has better convergence rate than those of the GA and $\mathrm{ABC}$ algorithm.

\subsection{Challenges}

The challenges of the existing works studied in the related works are presented in the section as follows:

- The estimation of the cost is the major issue that the researchers require to solve the issue [3]. The limitation of the traditional methods is about the appropriate statistical hypothesis testing that is not incorporated in the methods, which causes erroneous outcome for predictive accuracy [20].

- Comparison of the methods without the use of the statistical tests causes poor results. However, the researchers baseline their findings using the single indicators [23]. Most of the conventional methods face a lot of challenges that they never offered a comparative study using statistical hypothesis testing [15, 20].

- The estimation of the cost of the projects imposed huge tension among the project managers as they could not predict the cost accurately because of the standard metrics. They need comprehensive data, which are unavailable for all the initial projects, and it needs the experts in the particular domain to make the estimation accurately [8].

- The software cost is estimated using the clustering approach in $[15,16]$, which employed the unsupervised procedure, which stood inappropriate for the nonlinear software cost data. Moreover, these approaches are highly sensitive to the noisy environment.

\section{ORM-SCE: - proposed method of Optimal Regression model-based SCE using the WCO and regression model}

The paper proposes a novel optimization algorithm for developing an Optimal Regression model. The regression models used for the estimation of the software cost are the Kernel Logistic Regression model and the Linear Regression model. The project managers suffer a huge issue regarding the cost of the software. It has been reported that the estimation of the software cost consumes huge time and sometimes leads to human errors. To overcome these issues, the optimal cost estimation is carried out using the WCO algorithm, which aims at determining the optimal weights for the Linear Regression model and the Kernel Logistic Regression model.

Let us represent the software as $S$, which is subjected to the estimation of the cost based on a few parameters. If the cost considered is regarding the computational time, the software that possesses the minimum execution time is considered as the best software by the project managers. Let us represent the parameters used for estimating the cost of the software as

$$
A=\left\{A_{1}, A_{2}, \ldots, A_{k}, \ldots, A_{N}\right\}
$$

where $A_{k}$ indicates the $k^{\text {th }}$ parameter that estimates the cost of the software and $N$ is the total number of parameters present while estimating the cost of the software.

Figure 1 depicts a block diagram of the proposed method of optimal estimation of the software cost based on the parameters. Based on the parameters taken, the Linear Regression model and the Kernel Logistic Regression model predict the costs, such that the regression coefficients are optimally tuned using the proposed WCO algorithm.

\subsection{Linear Regression model}

The Linear Regression model is a high-latency model since it just needs to save the weights at the end of training and the space complexity is very low. The reason for choosing this model is that it is very easy to understand. Also, it is used for prediction problems, and it determines the best fit line, having a minimum error, between the dependent and independent variables. The Linear Regression model [30] explains the relationship between the dependent variable $Y$ and the independent variable $X$. In other words, it shows the deep analysis of the cost of the software based on the attributes used for estimating the cost of the software. It should be noted that the dependent variable $Y$, which is the cost of the software, should be continuous, but the independent variable $A_{k}$ can be either continuous or categorical. The output of the Linear Regression model is represented as

$$
Y^{L R}=X+\sum_{k=1}^{N} w_{k} \times A_{k}
$$

where $w_{k}$ denotes the regression coefficients and they are tuned optimally using the proposed WCO algorithm. Figure 2 shows a block diagram of the Linear Regression model, which takes the linear form by relating the inputs and the outputs. The output from the Linear Regression model is the cost of the software, which is computed using Eq. (2). The summation of the product of the regression weights and the attributes used to compute the software is summed with the regression line parameter $X$ to yield the 


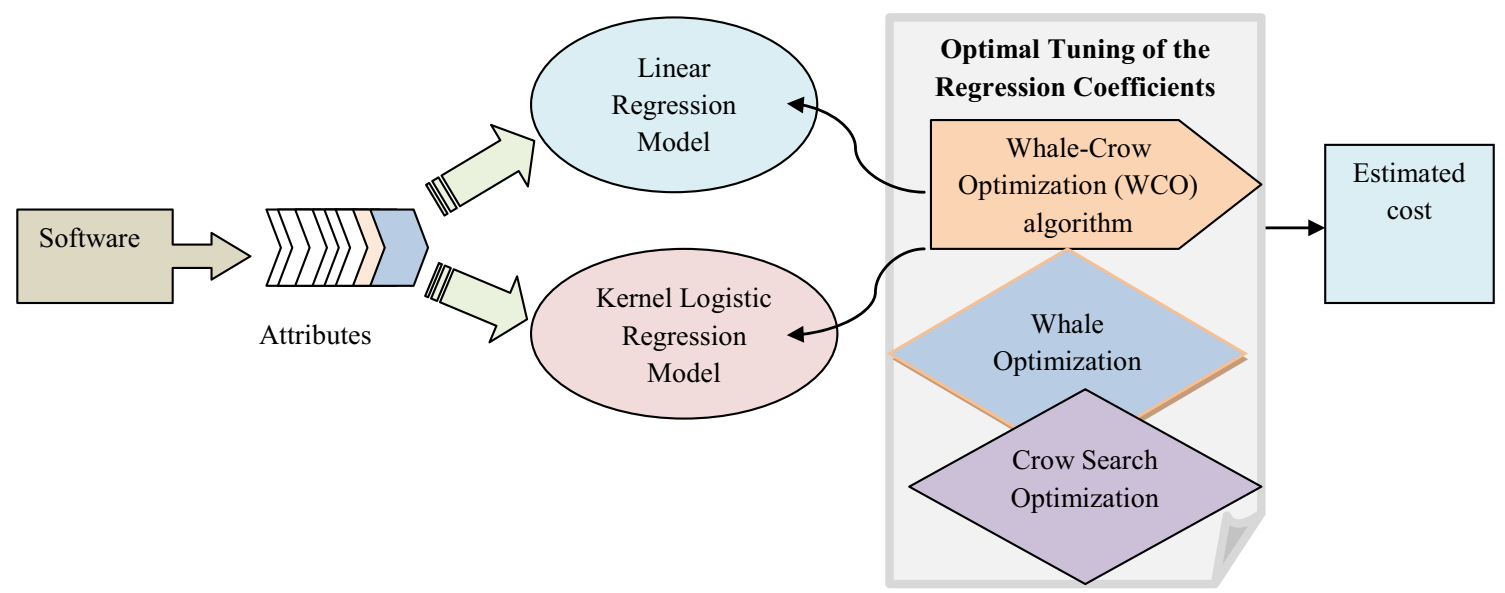

Figure 1. Block diagram of the proposed WCO-based regression model used for SCE.

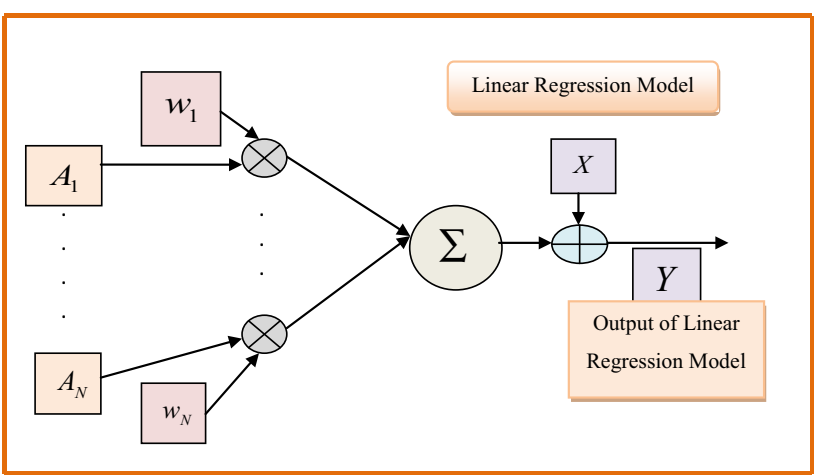

Figure 2. Block diagram of the Linear Regression model.

cost of the software. The regression coefficients are determined optimally using the proposed WCO algorithm.

\subsection{Kernel Logistic Regression model}

Kernel Logistic Regression [17] is a regression model used for evaluating the cost of the software through the analysis of the software based on the data attributes. The reason for using the Kernel Logistic Regression is that it has high classification performance, it provides class probabilities and it is an extension to multi-class classification. The output from the Kernel Logistic Regression model is determined using the following equation as

$$
Y^{K L R}=s+\sum_{k=1}^{N} \alpha_{k} \cdot K\left(A, A_{k}\right)
$$

where $A$ represents the parameter used for evaluating the execution cost, and $N$ is the total number of the parameters used for determining the cost of executing the software. $K\left(A, A_{k}\right)$ refers to the Kernel Regression function and the value of the Kernel Regression function is determined using

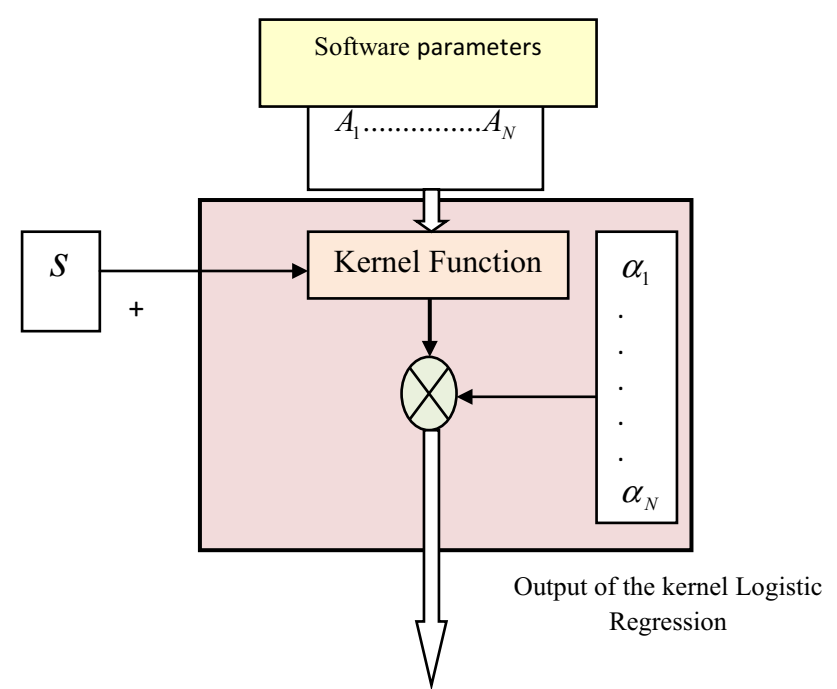

Figure 3. Schemata of the Kernel Logistic Regression model.

the greedy algorithm. The greedy algorithm [31] chooses the $q$ columns of the kernel matrix $K\left[\left(A_{k}, A_{g}\right)\right]_{N \times N}$ such that the span of these $q$ columns approximates the span of $K\left[\left(A_{k}, A_{g}\right)\right]_{N \times N}$ in the Frobenius norm. The regression coefficient is denoted as $\alpha_{k}$ and $k=N$. The value of the regression coefficient is optimally chosen using the proposed WCO algorithm. The Kernel Logistic Regression model uses the kernel function and the regression line coefficient to generate the cost of the software, as shown in figure 3 .

\subsection{Proposed WCO for the optimal selection of regression coefficients}

The proposed WCO is the integration of the standard CSA and the whale optimization algorithm (WOA), for the optimal computation of the regression coefficients. The 
main aim of the proposed WCO algorithm is to represent the single optimal solution enabling effective decision making in the evaluation of the software cost. The advantages of the proposed WCO algorithm are that the proposed algorithm relieves the burden of computational complexity and offers the best solution. The proposed WCO algorithm possesses an ability to converge to the global solution in the search space.

CSA [32] is based on the searching mechanism of the crows that live in the flock. The behaviours of the crow are referred to before the optimization process. The crow tends to memorize the position of the hiding places of the food inferred as an intelligent bird. Moreover, they follow the other crows brilliantly to execute the thievery, and they support their caches from being pilfered, which is based on an awareness probability. The main function of the awareness probability of the CSA is that it controls the intensification and diversification. The low value of the awareness probability indicates that the search of the CSA is performed at the local level, enabling the intensification, whereas the greater values of the awareness probability contribute to the exploration in the global level, enhancing the diversification. The main aim of the meta-heuristic algorithm is to obtain a balance between the diversification and the intensification, which is enabled in the CSA. Even though CSA presents interesting characteristics, its search strategy presents great difficulties when it faces high multimodal formulations. Hence, we integrate the WOA in the position update to improve the search process.

WOA [33] is based on the social behaviour of the humpback whales, which follow a special hunting mechanism termed as the bubble net method. The humpback whales generate the bubbles to encircle the prey and move towards them, and the hunting mechanism is carried out using three phases, namely the encircling phase, exploitation phase and the exploration phase. The existing optimization algorithms like PSO and ACO provide the best solution only in their path, and some of the iterative solutions are the candidate solutions. In addition, once a new population gets determined, the information of the previous iteration is lost. The advantages of WOA are the following: it is capable of exploring the search space globally; it explores the hot areas in the search space and in addition, it establishes a proper balance between the exploration process and the exploitation process.

The proposed WCO algorithm is the integration of both the CSA and the WOA to determine the regression coefficients.

3.3a Solution encoding of the proposed WCO algorithm: The solution encoding represents the solution to be obtained using the proposed WCO algorithm. The length of the solution depends on the total number of the regression coefficients present in the output equation of the Linear Regression method or the number of regression coefficients present in the Kernel Logistic Regression method. The number of the regression coefficients depends on the total number of parameters selected for evaluating the cost of the software. The dimension of the solution that holds the regression coefficients is denoted as $[1 \times N]$. The parameter $N$ is the total number of the regression coefficients used for evaluating the cost of the software.

3.3b Objective function: The main purpose of the objective function is to obtain the best software by estimating its cost. The parameters are used for determining the cost of the software and the methods used for estimating the cost of the software are the Linear Regression and the Kernel Logistic Regression. The Linear Regression and the Kernel Logistic Regression employ the MSE for estimating the cost of the software. The MSE, which is the error between the output $Y$ determined using the Linear Regression and its desired output, is determined using Eq. (4):

$$
M S E=\frac{1}{2}\left[Y-D^{*}\right]
$$

where $D^{*}$ is the desired cost of the software and $Y$ represents the output of Linear Regression and Kernel Logistic Regression models. The solution having minimum error will be selected as the best coefficient such that the cost can be predicted effectively. Similarly, in the case of the Kernel Logistic Regression, the MSE is the mean square of the kernel output and the desired cost.

3.3c WCO optimization algorithm: A detailed flowchart of the proposed WCO algorithm is depicted in figure 4. The initial population of the crow is initialized at the beginning of the proposed optimization process. The population is initialized randomly. The memory of the crow is updated for the total crow population, and the selection of the best memory is performed randomly for the first iteration. Whenever a crow intends to change the position to locate a new position of the hidden food, it follows the other crows and updates its position. If the new position of the crow is found to be feasible, the new position is kept, else the crow returns to its initial position. The fitness of the updated position is evaluated such that the position offering the minimum MSE is chosen as the best solution. While updating the new position of the crow, there are three conditions.

Condition 1: Whenever the position of the randomly chosen $j^{\text {th }}$ crow is greater than its awareness probability and when $(j=a)$, then the position update follows the whale optimization process. The parameter $a$ is the index of the best solution that offers the minimum value of the MSE.

Condition 2: Whenever the position of the randomly chosen $j^{\text {th }}$ crow exceeds its awareness probability and $(j \neq a)$, then the position update is based on the position update of the crow optimization process.

Condition 3: When the position of the randomly chosen $j^{\text {th }}$ crow is less than its awareness probability, then the position update is carried out randomly. This step happens in case of the infeasible solution. The detailed algorithmic steps of the proposed WCO algorithm are as follows. 


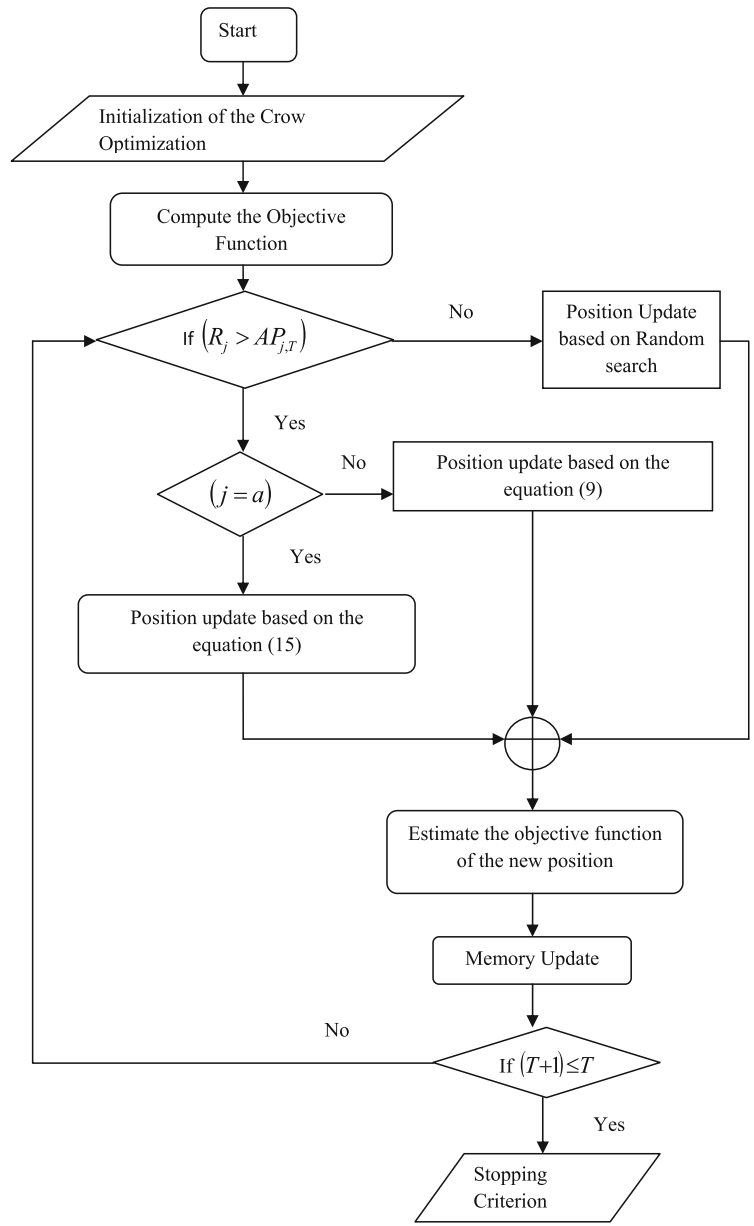

Figure 4. Flowchart of the proposed WCO algorithm.

Initialization: The fundamental step is the initialization of the total crow population in order to select the best position of the crow. The total population of the crow is presented as

$$
C=\left\{C_{1}, C_{2}, \ldots, C_{i}, \ldots C_{m}\right\}
$$

where $m$ is the total number of crows present in the population and $C_{i}$ represents the $i^{\text {th }}$ crow present in the population. The $m$ number of crows are distributed in the $l$ dimensional search area as the individual members of the flock, each corresponding to the feasible solution of the issue and there are $l$ number of decision variables. The position of the crows is denoted as

$$
\left[\begin{array}{ccccc}
Z_{1}^{1} & Z_{2}^{1} & \cdot & \cdot & Z_{l}^{1} \\
Z_{1}^{2} & Z_{2}^{2} & \cdot & \cdot & Z_{1}^{2} \\
\cdot & \cdot & \cdot & \cdot & \cdot \\
\cdot & \cdot & \cdot & \cdot & \cdot \\
Z_{1}^{m} & Z_{2}^{m} & \cdot & \cdot & Z_{l}^{m}
\end{array}\right] .
$$

The memory of the crow is given by

$$
\left[\begin{array}{ccccc}
r_{1}^{1} & r_{2}^{1} & \cdot & \cdot & r_{l}^{1} \\
r_{1}^{2} & r_{2}^{2} & \cdot & \cdot & r_{1}^{2} \\
\cdot & \cdot & \cdot & \cdot & \cdot \\
\cdot & \cdot & \cdot & \cdot & \cdot \\
r_{1}^{m} & r_{2}^{m} & \cdot & \cdot & r_{l}^{m}
\end{array}\right] .
$$

Initially, at the beginning of the optimization, the crow does not know the hidden food and the positions of the hidden food are considered to be in their initial positions. All the crows hold the memory of the hidden food and the memory of the hidden food for all the crows present in the flock is formulated in Eq. (7).

Evaluation of the objective function: The optimal selection of the best position of memory is based on the fitness measure that depends on the MSE, and the value of the MSE should be a minimum value. The objective of the Linear Regression coefficient aims towards the minimum value of the MSE, and the position of the crow that corresponds to the minimum value of MSE is selected as the best solution. Similarly, Kernel Logistic Regression uses the objective function that aims at the minimization of the kernel constraint for the selection of the optimal position of the crow, and the kernel constraint is based on the MSE. In other words, the Kernel Logistic Regression and the Linear Regression are the methods employed for the evaluation of the cost of the software in order to choose the software that requires minimum execution cost. The objective function in terms of MSE is shown in Eq. (4).

Obtain the new position of the crow using the proposed WCO algorithm: The position of the crow is updated based on the proposed WCO algorithm that comprises three conditions. Initially, whenever the $i^{\text {th }}$ crow intends to update the position, the $i^{\text {th }}$ crow opts any crow from the population and follows that crow to discover the location of the hidden objects. Let us denote the predecessor crow as $C_{j}$ and the $i^{\text {th }}$ crow follows the $j^{\text {th }}$ crow in order to update the position. It is essential to validate the feasibility of the newly generated positions such that if the position is feasible, the position is updated based on the Eq. (16) or (10). In the case of a non-feasible solution, the crow remains in its initial position or otherwise, position update takes place accordingly. If the position of the $j^{\text {th }}$ crow is greater than the awareness probability and if the position of the $j^{\text {th }}$ crow is the global best position, the position update of the $i^{\text {th }}$ crow is performed based on Eq. (16). Otherwise, the position update of the $i^{\text {th }}$ crow depends on Eq. (10). If the awareness probability exceeds the position of the $j^{\text {th }}$ crow and if the position of the $j^{\text {th }}$ crow is the local best solution, the position update of the $i^{\text {th }}$ crow is performed randomly.

The initial condition is the following: when the random number of the $j^{\text {th }}$ crow exceeds the awareness of the $j^{\text {th }}$ crow, the position update performed is based on the WOA. The range of the random number varies between 0 and 1 . The WOA is modified with the CSA, and the equation of the WOA is shown in Eq. (8). The position update equation 
of the WOA is shown in Eq. (10) for obtaining a new equation such that the newly developed position update equation of the proposed WCO converges quickly to the global optimal solution and memorizes the optimal position for searching the hidden food in the forthcoming search mechanisms. The position update in the WOA is as follows:

$$
\vec{Z}(T+1)=\overrightarrow{Z^{*}}(T)-\vec{B} \cdot \vec{Q}
$$

where $\vec{B}$ and $\vec{d}$ are the coefficient vectors, $\vec{Q}$ is the distance vector and $Z^{*}$ is the position vector of the best solution. Rearranging Eq. (8), we get

$$
\overrightarrow{Z^{*}(T)}=\vec{Z}(T+1)+\vec{B} \cdot \vec{Q}
$$

The position update equation of the $i^{\text {th }}$ crow using the CSA is

$$
Z^{i, T+1}=Z^{i, T}+R_{i} * L^{i, T} *\left(Z^{*}-Z^{i, T}\right) .
$$

Substituting Eq. (9) in Eq. (10) gives

$$
\begin{aligned}
& Z^{i, T+1}= Z^{i, T}+R_{i} * L^{i, T} *\left[\left(Z^{i, T+1}+\vec{B} \cdot \vec{Q}\right)-Z^{i, T}\right] \\
& Z^{i, T+1}=Z^{i, T}+R_{i} * L^{i, T} *\left[Z^{i, T+1}+\vec{B} \cdot \vec{Q}-Z^{i, T}\right] \\
& Z^{i, T+1}=Z^{i, T}+R_{i} * L^{i, T} * Z^{i, T+1}+R_{i} * L^{i, T} * \vec{B} \cdot \vec{Q}-R_{i} \\
& * L^{i, T} * Z^{i, T}
\end{aligned}
$$

$$
\begin{aligned}
Z^{i, T+1}-R_{i} * L^{i, T} * Z^{i, T+1}= & Z^{i, T}+R_{i} * L^{i, T} * \vec{B} \cdot \vec{Q}-R_{i} \\
& * L^{i, T} * Z^{i, T}
\end{aligned}
$$

$$
Z^{i, T+1}\left(1-R_{i} * L^{i, T}\right)=Z^{i, T}\left(1-R_{i} * L^{i, T}\right)+R_{i} * L^{i, T} * \vec{B} \cdot \vec{Q}
$$

$$
Z^{i, T+1}=\frac{1}{\left(1-R_{i} * L^{i, T}\right)}\left[Z^{i, T}\left(1-R_{i} * L^{i, T}\right)+R_{i} * L^{i, T} * \vec{B} \cdot \vec{Q}\right]
$$

where $\vec{B}=2 \vec{b} \cdot \vec{R}-\vec{b}, \vec{Q}=\left|\vec{d} \cdot Z^{*}(T)-Z^{i, T}\right|$ and $\vec{d}=$ 2. $\vec{R}$ where $R$ is a random number, $L^{i}$ denotes the flight length of the $i^{\text {th }}$ crow and $Z^{*}(T)$ indicates the best solution of the optimization process. The value of random number $R$ varies in the range $0 \leq R \leq 1$ during exploitation and exploration. The coefficient vectors are denoted as $\vec{B}$ and $\vec{Q}$, I I refers to the absolute value and ' ' indicates the element-wise multiplication. The value of $\vec{b}$ is linearly decreased from 2 to 0 .

Evaluate the objective function of the newly generated position of the crows: The objective function is checked for the newly updated position of the $i^{\text {th }}$ crow for the selection of the best solution.

Memory updation of the crow: The memory of the newly updated position is evaluated based on the following formula (the main function of memorizing is to remember the availability of the hidden food for future search mechanisms):

$$
r^{i, T+1}=\left\{\begin{array}{cc}
Z^{i, T+1} ; & f\left(Z^{i, T+1}\right) \\
Z^{i, T} ; & \text { is better than } f\left(Z^{i, T}\right) \\
\text { Otherwise }
\end{array}\right.
$$

where the function $f()$ is the fitness measure to determine the best solution. It is clear that the position of the $i^{\text {th }}$ crow is updated only when the new position is found to be better when compared with the initial position of the $i^{t h}$ crow. This estimation is based on the fitness, and the crow updates its position based upon the minimum value of the fitness for the new position or else the crow drops the position update to stay in its initial position.

Stopping criterion: The steps from updating the new position until the memory update are repeated until the maximum iteration is reached. Finally, the best position of the crow is retrieved based on the objective function.

Therefore, the regression coefficients are optimally tuned for both the Linear Regression method and the Kernel Logistic Regression method so that the cost of the software is determined optimally. The software that offers the minimum cost is chosen so that this method relieves the burden of the project managers in estimating the cost of the software.

\section{Results and discussion}

This section presents the results and a discussion of the proposed method, and comparative analyses with the existing methods are made to prove the effectiveness of the proposed method.

\subsection{Experimental set-up}

Experimentation of the proposed technique of SCE is done in a system with 2 GB RAM, Intel core processor and Windows 10 Operating System. The proposed method of cost estimation is implemented in the JAVA tool.

\subsection{Dataset description}

The experimentation is carried out using four datasets: cocomo81 dataset, cocomonasa_2 dataset, cocomonasa_v1 dataset and Desharnais dataset. The datasets cocomonasa_v1, cocomonasa_2, Desharnais and cocomo81 are taken from the Promise software engineering repository [34]. The cocomo81 dataset comprises 63 instances and 17 data attributes used for SCE. The Desharnais dataset comprises 81 instances and 12 attributes, and cocomonasa_2 consists 
of 93 instances and 24 attributes. The cocomonasa_v1 comprises 60 instances and 17 attributes.

\subsection{Performance metrics}

The metric employed for the evaluation of the SCE methods is the mean magnitude of relative error (MMRE). The main purpose of MMRE is to select the best model for estimating the software cost. It is defined as the ratio of the difference between the actual cost and the estimated cost to the actual cost. The MMRE is given as,

$$
\text { MMRE }=\frac{\text { actual cost }- \text { prediction cost }}{\text { actual cost }} .
$$

\subsection{Competing methods}

The methods taken for comparison include the Linear Regression [30], Kernel Regression [17], CSA + Linear Regression [32] and CSA + Kernel Regression [32], which are compared with the proposed methods $\mathrm{WCO}+$ Linear Regression, WCO + Kernel Regression, PSO-FCM [35], Tabu Search + GA [36] and PSOCP [37], to prove the superiority of the proposed methods.

\subsection{Performance analysis using the training and the testing data}

This section depicts the performance analysis of the proposed method using four datasets employed for training and testing. Figure 5 depicts the performance analysis using the training data with varying population sizes, denoted as $P$. Figure 5(a) shows the analysis using the cocomo81 dataset. The analysis is carried out in terms of the MMRE, and the value of the MMRE should be minimum. The analysis depends on the population sizes 10, 20 and 30. For the training percentage of $90 \%$, the MMRE rate is found to be $0.3894,0.5870,0.3894,0.3333,0.5867$ and 0.3901 for $\mathrm{WCO}+$ Linear Regression and WCO + Kernel Regression at the varying population sizes of 10, 20 and 30, respectively. Figure 5(b) shows the analysis using the cocomonasa_2 dataset. The analysis is carried out in terms of the MMRE, and the value of the MMRE should be a minimum. The analysis depends on the population sizes 10 , 20 and 30. For the training percentage of $90 \%$, the MMRE rate is found to be $0.3975,0.2704,0.3975,0.3964,0.3975$ and 0.3964 , respectively, for WCO + Linear Regression and $\mathrm{WCO}+$ Kernel Regression at the varying population sizes of 10, 20 and 30. Figure 5(c) shows the analysis using the cocomonasa_v1dataset. The analysis is carried out in terms of the MMRE, and the value of the MMRE should be a minimum. For the training percentage of $90 \%$, the MMRE rate is found to be $0.1482,0.3333,0.3226,0.1981,0.3226$
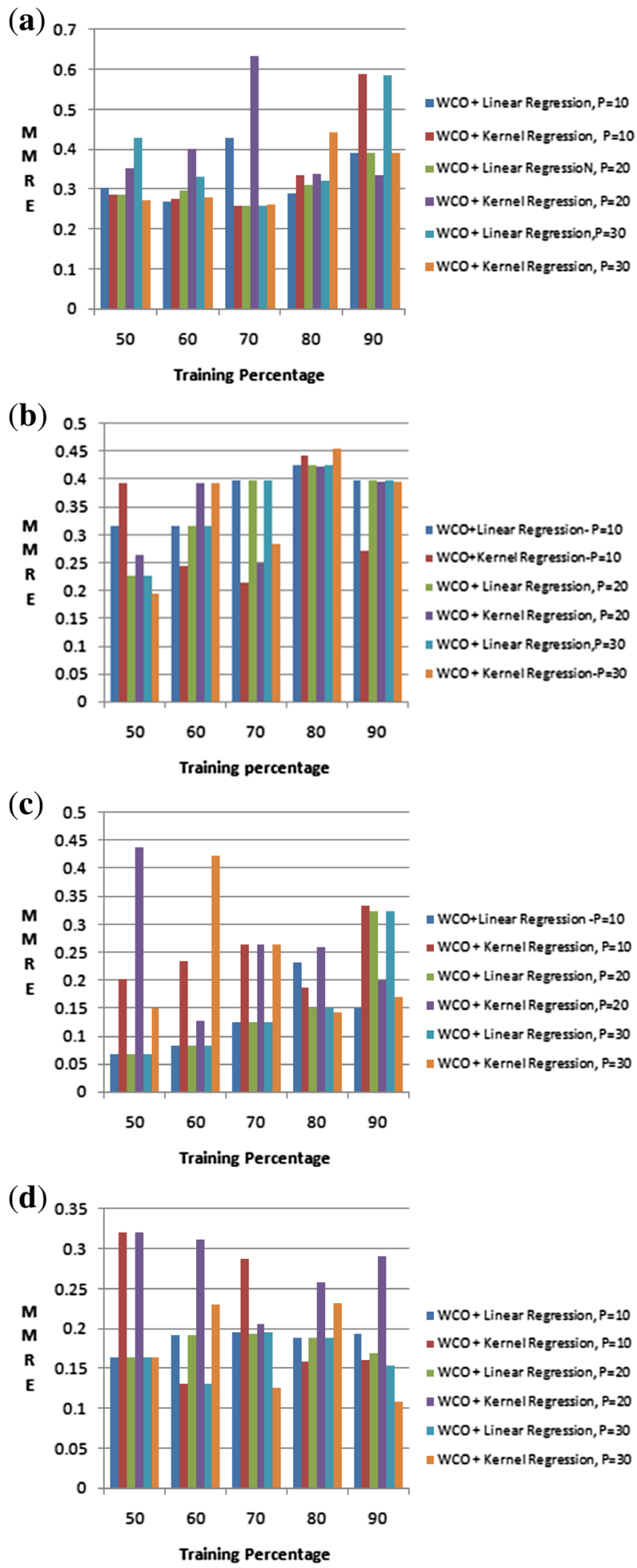

Figure 5. Performance analysis using the training data of (a) cocomo81, (b) cocomonasa_2, (c) cocomonasa_v1 and (d) desharnais.

and 0.1683 , respectively, for $\mathrm{WCO}+$ Linear Regression and $\mathrm{WCO}+$ Kernel Regression at the population sizes fixed at 10, 20 and 30. Figure 5(d) shows the analysis using the desharnais dataset. The analysis is carried out in terms 
of the MMRE and the value of the MMRE should be a minimum. The analysis depends on the population sizes 10 , 20 and 30. For the training percentage of $90 \%$, the MMRE rate is found to be $0.1933,0.1610,0.1684,0.2908,0.1525$ and 0.1085 for $\mathrm{WCO}+$ Linear Regression and $\mathrm{WCO}+$ Kernel Regression at the varying population sizes of 10,20 and 30, respectively. It is clear from the figure that with the increase in the training percentages, the MMRE rate increases.

Figure 6 depicts the performance analysis using the testing data. Figure 6(a) shows the analysis using the cocomo81 dataset. The analysis is carried out in terms of the MMRE, and the value of the MMRE should be a minimum. The analysis depends on the population sizes 10 , 20 and 30. For the training percentage of 90\%, the MMRE rate is found to be $0.3582,0.5906,0.3582,0.3333,0.5895$ and 0.3601 , respectively, for $\mathrm{WCO}+$ Linear Regression and $\mathrm{WCO}+$ Kernel Regression at the varying population sizes of 10, 20 and 30, respectively. Figure 6(b) shows the analysis using the cocomonasa_2 dataset. The analysis is carried out in terms of the MMRE, and the value of the MMRE should be a minimum. For the training percentage of $90 \%$, the MMRE rate is found to be 0.4929, 0.3906, $0.4929,0.4407,0.4929$ and 0.5117 , respectively, for $\mathrm{WCO}+$ Linear Regression and $\mathrm{WCO}+$ Kernel Regression at the varying population sizes of 10,20 and 30 . Figure 6(c) shows the analysis using the cocomonasa_v1 dataset. The analysis is carried out in terms of the MMRE, and the value of the MMRE should be a minimum. For the training percentage of $90 \%$, the MMRE rate is found to be $0.1110,0.3333,0.1110,0.2330,0.1103$ and 0.1683 , respectively, for $\mathrm{WCO}+$ Linear Regression and $\mathrm{WCO}+$ Kernel Regression at the varying population sizes of 10, 20 and 30. Figure 6(d) shows the analysis using the desharnais dataset in terms of the MMRE. For the training percentage of $90 \%$, the MMRE rate is found to be 0.0732 , $0.1610,0.0732,0.2911,0.0732$ and 0.2307 , respectively, for WCO + Linear Regression and WCO + Kernel Regression at the varying population sizes of 10, 20 and 30. It is clear from the figure that with the increase in the training percentages, the MMRE rate increases.

\subsection{Comparative analysis}

This section depicts the comparative analysis of the proposed method using four datasets employed for training and testing. Figure 7 depicts the performance analysis using the training data. Figure 7(a) shows the analysis using the cocomo81 dataset. The analysis is carried out in terms of the MMRE, and the value of the MMRE should be a minimum for the effective method. For the training percentage of $90 \%$, the MMRE rate is found to be 2.5421, $10.2705,0.8401,0.3333,0.4267,0.4826,0.3478,0.2415$ and 0.2434 , respectively, for Linear Regression, Kernel Regression, CSA + Linear Regression, CSA + Kernel (a)

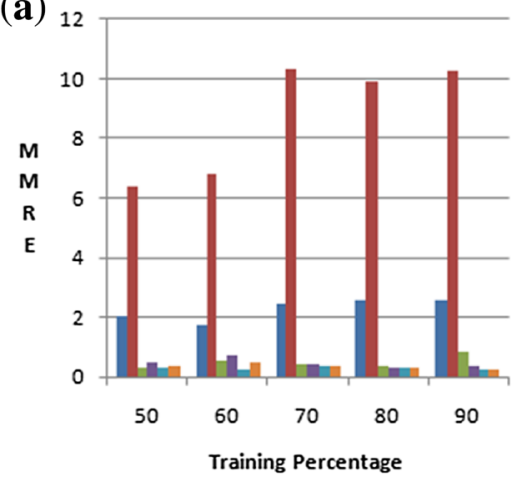

- Linear Regression

- Kernel Regression

m CSA + Linear Regression

nCSA + Kernel Regression

- $W C O+$ Linear Regression

- $\mathrm{WCO}+$ Kernel Regression

(b)

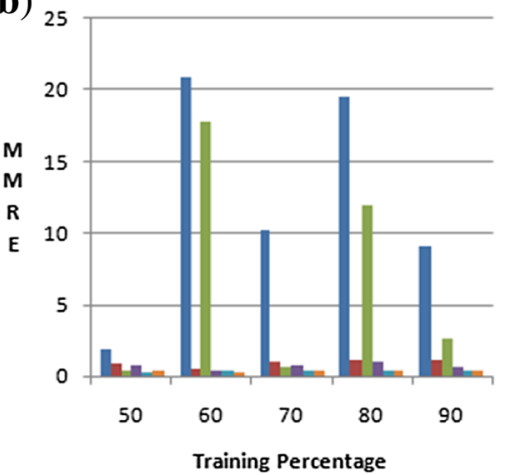

- Linear Regression

- Kernel Regression

a CSA + Linear Regression

- CSA + Kernel Regression

- $\mathrm{WCO}+$ Linear Regression

a $\mathrm{WCO}$ + Kernel Regression

(c)

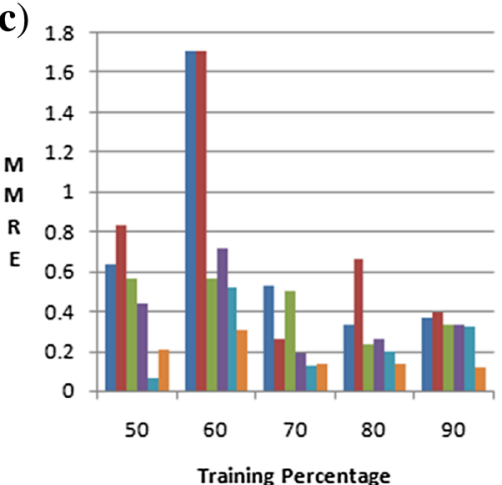

E Linear Regression

- Kernel Regression

a CSA + Linear Regression

a CSA + Kernal Regression

- $\mathrm{WCO}+$ Linear Regression

w $W C O+$ Kernel Regression

(d)

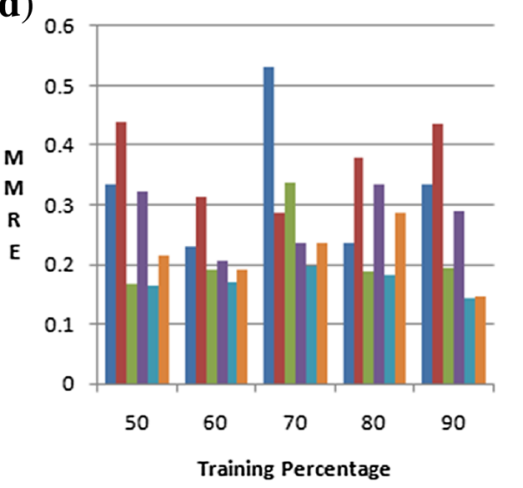

- Linear Regression

- Kernel Regression

a CSA + Linear Regression

n CSA + Kernel Regression

$w C O+$ Linear Regression

- $\mathrm{WCO}+$ Kernel Regression

Figure 6. Performance analysis using the testing data of (a) cocomo81, (b) cocomonasa_2, (c) cocomonasa_v1 and (d) desharnais.

Regression, PSO-FCM, Tabu Search + GA, PSOCP, $\mathrm{WCO}+$ Linear Regression and $\mathrm{WCO}+$ Kernel Regression. Figure 7(b) shows the analysis using the 
(a)

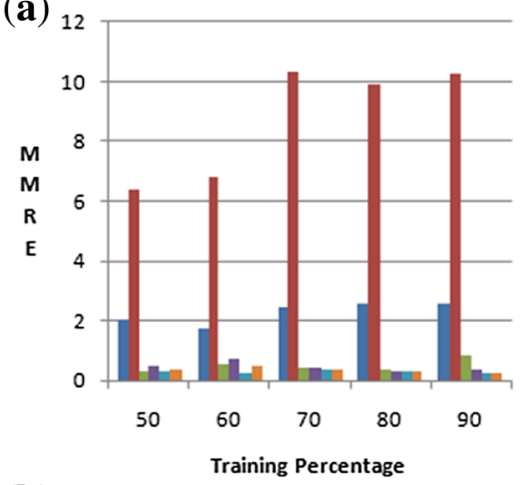

(b)

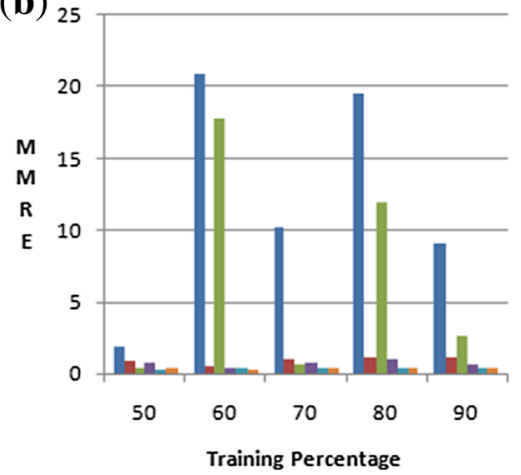

(c)

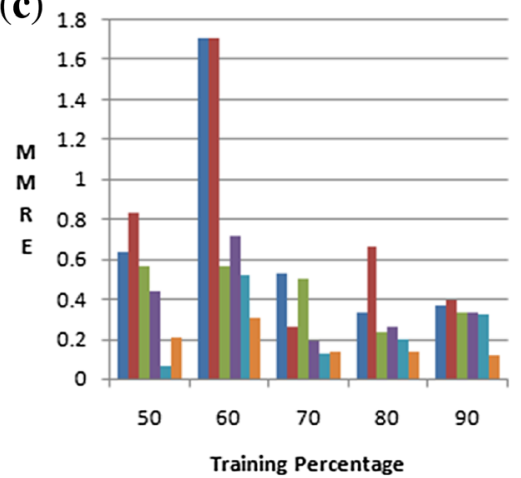

(d)

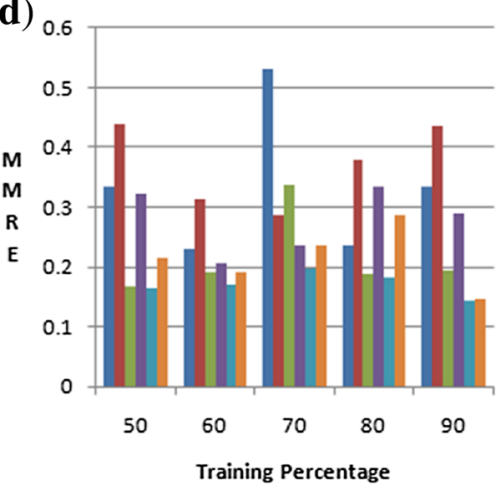

- Linear Regression

- Kernel Regression

m CSA + Linear Regression

n CSA + Kernel Regression

WCO + Linear Regression

a $\mathrm{WCO}+$ Kernel Regression

n Linear Regression

nernel Regression

I CSA + Linear Regression

a CSA + Kernel Regression

a $W \mathrm{CO}+$ Linear Regression

w $\mathrm{WCO}+$ Kernel Regression

- Linear Regression

- Kernel Regression

= CSA + Linear Regression

- CSA + Kernal Regression

- $W C O+$ Linear Regression

wCO + Kernel Regression

- Linear Regression

- Kernel Regression

= CSA + Linear Regression

- CSA + Kernel Regression

- $W C O+$ Linear Regression

w $\mathrm{WCO}+$ Kernel Regression

Figure 7. Comparative analysis using the training data of (a) cocomo81, (b) cocomonasa_2, (c) cocomonasa_v1 and (d) desharnais.

cocomonasa_2 dataset. The analysis is carried out in terms of the MMRE, and the value of the MMRE should be a minimum. For the training percentage of $90 \%$, the MMRE

rate is found to be $9.1064,1.1234,2.5610,0.5733,0.5874$, 0.4695, 0.4721, 0.3975 and 0.3964, respectively, for Linear Regression, Kernel Regression, CSA + Linear Regression, CSA + Kernel Regression, PSO-FCM, Tabu Search + GA, PSOCP, WCO + Linear Regression and $\mathrm{WCO}+$ Kernel Regression. Figure 7(c) shows the analysis using the cocomonasa_v1dataset, carried out in terms of the MMRE and the value of the MMRE should be a minimum. For the training percentage as $90 \%$, the MMRE rate is found to be $0.3682,0.3922,0.3333,0.3333,0.3951,0.3581,0.3345$, 0.3226 , and 0.1188 respectively for Linear Regression, Kernel Regression, CSA + Linear Regression, CSA + Kernel Regression, PSO-FCM, Tabu Search + GA, PSOCP, WCO + Linear Regression and WCO + Kernel Regression, respectively. Figure 7(d) shows the analysis using the desharnais dataset. The analysis is carried out in terms of the MMRE, and the value of the MMRE should be a minimum. For the training percentage of $90 \%$, the MMRE rate is found to be $0.3333,0.4344,0.1933,0.2908,0.1864$, $0.1654,0.1954,0.1425$ and 0.1467 , respectively, for Linear Regression, Kernel Regression, CSA + Linear Regression, CSA + Kernel Regression, PSO-FCM, Tabu Search + GA, PSOCP, WCO + Linear Regression and $\mathrm{WCO}+$ Kernel Regression.

Figure 8(a) shows the analysis using the cocomo81 dataset. The analysis is carried out in terms of the MMRE, and the value of the MMRE should be a minimum for the effective method. For the training percentage of $90 \%$, the MMRE rate is found to be 1.9994, 9.6781, 0.8458, 0.3333, $0.2658,0.2567,0.2517,0.2415$ and 0.2434 , respectively, for Linear Regression, Kernel Regression, CSA + Linear Regression, CSA + Kernel Regression, PSO-FCM, Tabu Search + GA, PSOCP, WCO + Linear Regression and $\mathrm{WCO}+$ Kernel Regression, respectively. Figure 8(b) shows the analysis using the cocomonasa_2 dataset. The analysis is carried out in terms of the MMRE, and the value of the MMRE should be a minimum. For the training percentage of $90 \%$, the MMRE rate is found to be 24.6083, $1.5709,2.5610,0.5733,0.5421,0.5348,0.5264,0.4929$ and 0.5117 for Linear Regression, Kernel Regression, CSA + Linear Regression, CSA + Kernel Regression, PSO-FCM, Tabu Search + GA, PSOCP, WCO + Linear Regression and $\mathrm{WCO}+$ Kernel Regression, respectively. Figure 8(c) shows the analysis using the cocomonasa_v1dataset. For the training percentage of 90\%, the MMRE rate is found to be $0.3686,0.3538,0.3333,0.3333,0.1256$, $0.1546,0.1462,0.1110$ and 0.1188 , respectively, for Linear Regression, Kernel Regression, CSA + Linear Regression, CSA + Kernel Regression, PSO-FCM, Tabu Search + GA, PSOCP, WCO + Linear Regression and $\mathrm{WCO}+$ Kernel Regression. Figure 8(d) shows the analysis using the desharnais dataset. The analysis is carried out in terms of the MMRE and the value of the MMRE should be a minimum. For the training percentage of $90 \%$, the MMRE rate is found to be $0.3333,0.4203,0.1425,0.2911,0.1689$, $0.1657,0.15632,0.0732$ and 0.1467 , respectively, for 

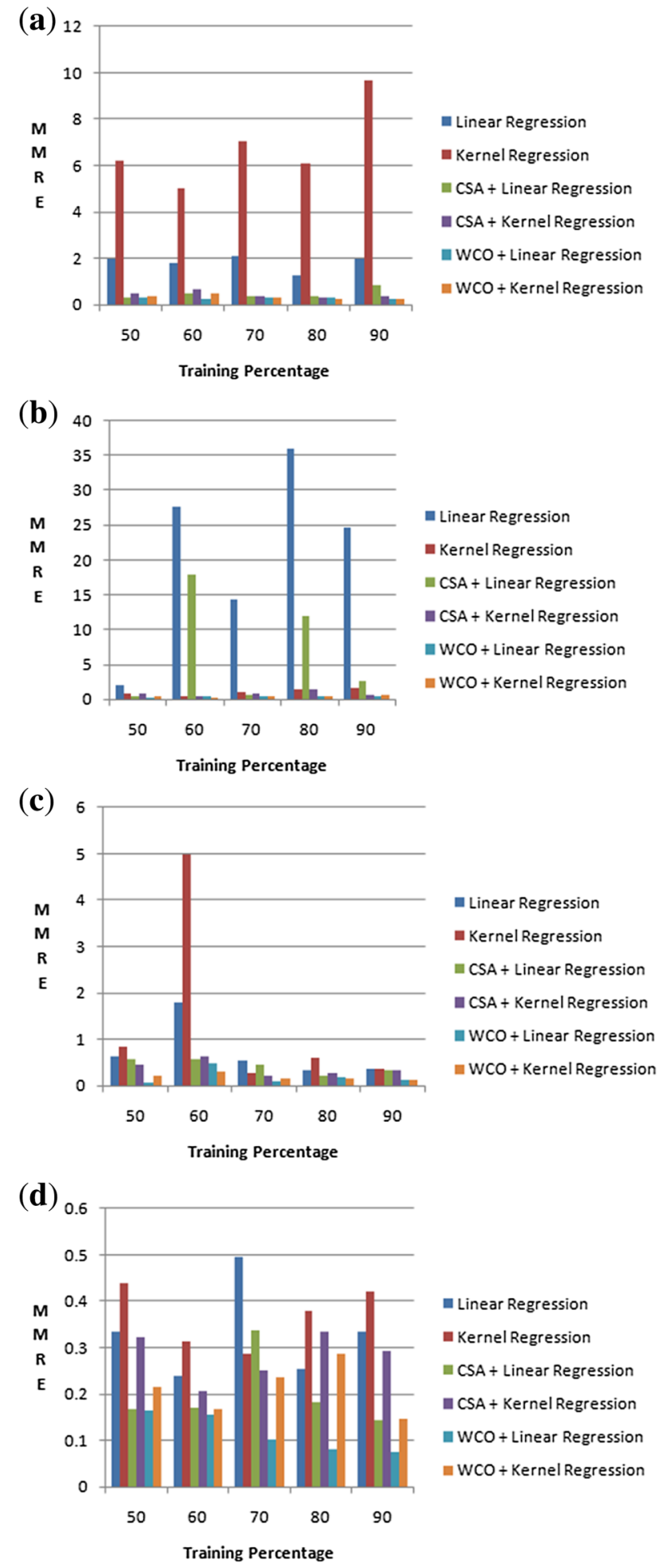

Figure 8. Comparative analysis using the testing data of (a) cocomo81, (b) cocomonasa_2, (c) cocomonasa_v1 and (d) desharnais.

Linear Regression, Kernel Regression, CSA + Linear Regression, CSA + Kernel Regression, PSO-FCM, Tabu Search + GA, PSOCP, WCO + Linear Regression and $\mathrm{WCO}+$ Kernel Regression.

\subsection{Convergence analysis}

Figure 9 shows the convergence analysis graph based on the objective function. The analysis is performed by varying the iteration from 0 to 100 . The analysis using the four datasets, cocomo81, cocomonasa_2, cocomonasa_v1 and desharnais, is shown in figure 9(a), (b), (c) and (d), respectively.

Figure 10 shows the convergence analysis graph based on the distance. The analysis is performed by varying the iteration from 0 to 100 . The analysis using the four datasets, cocomo81, cocomonasa_2, cocomonasa_v1 and desharnais, is shown in figure 10(a), (b), (c) and (d), respectively.

\subsection{Comparative discussion}

The comparative analysis using cocomo81dataset is highlighted in table 1 in terms of the best MMRE. The proposed method attained the MMRE of 0.2435 for Kernel Regression and 0.2416 for Linear Regression using the training data and 0.2435 for Kernel Regression and 0.2416 for Linear Regression using the testing data. The other methods, PSO-FCM, Tabu Search + GA, PSOCP, the Linear Regression and the Kernel Regression, yield a greater value when compared with the optimization-based regressions. Thus, it is obvious from table 1 that the MMRE rate is low for the optimization algorithms, and the proposed method outperformed all the methods.

The comparative analysis using cocomonasa_2 is highlighted in table 2 in terms of the minimum MMRE. The proposed method attained the MMRE of 0.2449 for Kernel Regression and 0.2274 for Linear Regression using the training data and 0.2695 for Kernel Regression and 0.2363 for Linear Regression using the testing data. The other methods have a high MMRE rate when compared with the proposed method.

The comparative analysis using cocomonasa_v1 is highlighted in table 3 in terms of the minimum MMRE. The proposed method attained the MMRE of 0.1188 for Kernel Regression and 0.0667 for Linear Regression using the training data and 0.1188 for Kernel Regression and 0.0667 for Linear Regression using the testing data. From table 3, it can be shown that the proposed method has the minimum MMRE rate when compared with the other comparative methods.

The comparative analysis using desharnais is highlighted in table 4 in terms of the minimum MMRE. The proposed method attained the MMRE of 0.1468 for Kernel Regression and 0.1425 for Linear Regression using the training data and 0.1468 for Kernel Regression and 0.0733 for Linear Regression using the testing data. From table 4, it can be shown that the proposed method has the minimum MMRE rate when compared with the other comparative methods. 

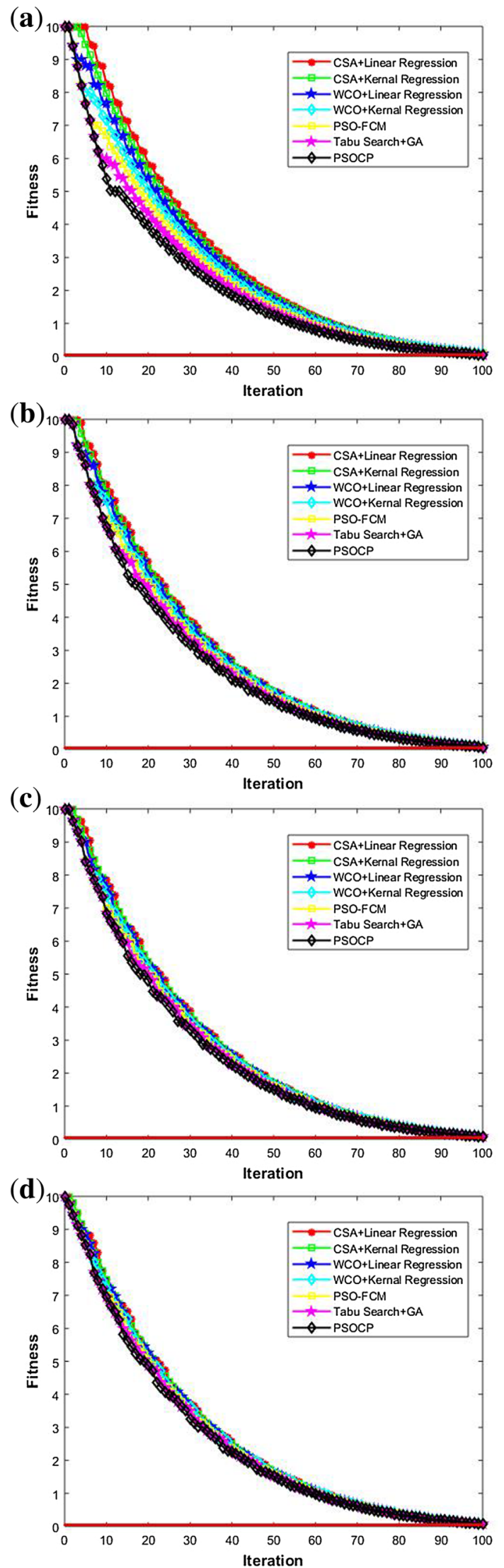

Figure 9. Convergence analysis based on objective function: (a) cocomo81, (b) cocomonasa_2, (c) cocomonasa_v1 and (d) desharnais.
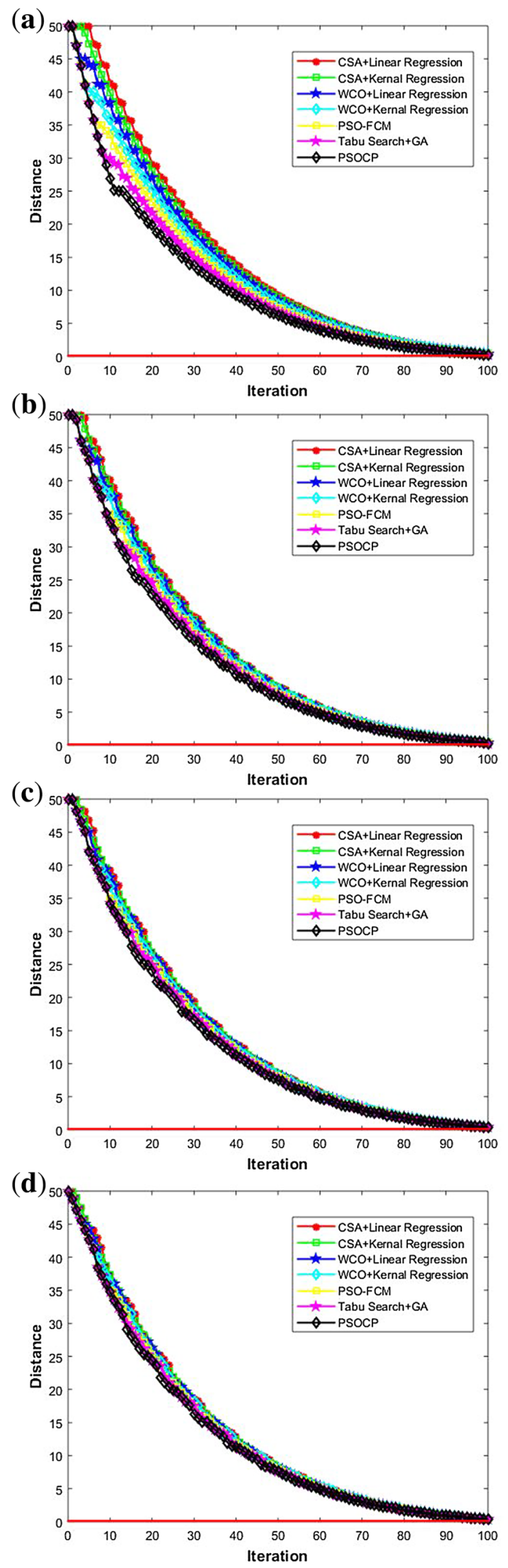

Figure 10. Convergence analysis based on distance: (a) cocomo81, (b) cocomonasa_2, (c) cocomonasa_v1 and (d) desharnais. 
Table 1. Comparative analysis of the competing methods using cocomo81.

\begin{tabular}{lcc}
\hline & $\begin{array}{c}\text { Training } \\
\text { data }\end{array}$ & $\begin{array}{c}\text { Testing } \\
\text { data }\end{array}$ \\
\hline Linear Regression & 1.7097 & 1.2621 \\
Kernel Regression & 6.3925 & 4.9962 \\
CSA + Linear Regression & 0.2850 & 0.2805 \\
CSA + Kernel Regression & 0.3231 & 0.3231 \\
PSO-FCM & 0.4267 & 0.2658 \\
Tabu Search + GA & 0.3957 & 0.2567 \\
PSOCP & 0.3478 & 0.2517 \\
Proposed WCO + Linear & 0.2416 & 0.2416 \\
$\quad$ Regression & & \\
Proposed WCO + Kernel & 0.2435 & 0.2435 \\
Regression & & \\
\hline
\end{tabular}

Table 2. Comparative analysis of the competing methods using cocomonasa_2.

\begin{tabular}{lcc}
\hline & $\begin{array}{c}\text { Training } \\
\text { data }\end{array}$ & $\begin{array}{c}\text { Testing } \\
\text { data }\end{array}$ \\
\hline Linear Regression & 1.9089 & 1.9089 \\
Kernel Regression & 0.4457 & 0.4457 \\
CSA + Linear Regression & 0.3333 & 0.3333 \\
CSA + Kernel Regression & 0.3922 & 0.4108 \\
PSO-FCM & 0.4589 & 0.3912 \\
Tabu Search + GA & 0.4623 & 0.3895 \\
PSOCP & 0.3981 & 0.3945 \\
Proposed WCO + Linear & 0.2274 & 0.2363 \\
$\quad$ Regression & & \\
Proposed WCO + Kernel & 0.2449 & 0.2695 \\
$\quad$ Regression & & \\
\hline
\end{tabular}

Table 3. Comparative analysis of the competing methods using cocomonasa_v1.

\begin{tabular}{lcc}
\hline & $\begin{array}{c}\text { Training } \\
\text { data }\end{array}$ & $\begin{array}{c}\text { Testing } \\
\text { data }\end{array}$ \\
\hline Linear Regression & 0.3274 & 0.3274 \\
Kernel Regression & 0.2633 & 0.2709 \\
CSA + Linear Regression & 0.2324 & 0.2033 \\
CSA + Kernel Regression & 0.1879 & 0.2049 \\
PSO-FCM & 0.1526 & 0.1256 \\
Tabu Search + GA & 0.1624 & 0.1546 \\
PSOCP & 0.1489 & 0.1462 \\
Proposed WCO + Linear & 0.0667 & 0.0667 \\
$\quad$ Regression & & \\
Proposed WCO + Kernel & 0.1188 & 0.1188 \\
$\quad$ Regression & & \\
\hline
\end{tabular}

Table 4. Comparative analysis of the competing methods using desharnais.

\begin{tabular}{lcc}
\hline & $\begin{array}{c}\text { Training } \\
\text { data }\end{array}$ & $\begin{array}{c}\text { Testing } \\
\text { data }\end{array}$ \\
\hline Linear Regression & 0.2301 & 0.2401 \\
Kernel Regression & 0.2868 & 0.2879 \\
CSA + Linear Regression & 0.1663 & 0.1425 \\
CSA + Kernel Regression & 0.2062 & 0.2062 \\
PSO-FCM & 0.1864 & 0.1689 \\
Tabu Search + GA & 0.1654 & 0.1657 \\
PSOCP & 0.1954 & 0.1563 \\
Proposed WCO + Linear & 0.1425 & 0.0733 \\
$\quad$ Regression & & \\
Proposed WCO + Kernel & 0.1468 & 0.1468 \\
$\quad$ Regression & & \\
\hline
\end{tabular}

\section{Conclusion}

The paper uses an optimization algorithm in order to estimate the Optimal Regression coefficients employed in the SCE methods. This paper uses the Linear Regression model and the Kernel Logistics Regression model to estimate the cost of the software. The Linear Regression model gains importance in terms of the linear relationship that exists between the cost of the software and the attributes employed for estimating the cost of the software. The Linear Regression model, which is a linear combination of the linear coefficients or the software attributes, is effective in determining the cost of the software when optimization is used to determine the optimal coefficients. The need for the regression coefficient is that it is essential to determine the cost using the optimization process. The kernel functions are employed in the Kernel Regression. Thus, the proposed WCO algorithm aims at finding the optimal coefficients for the regression models such that the cost of the software is determined effectively. The experimentation performed using the datasets taken from the Promise repository proves that the proposed method yields a better value of MMRE with a value of 0.2442 when compared with the existing methods and the proposed method. In future, deep learning methods will be used for estimating the software cost.

\section{References}

[1] Kaushik A, Soni A K and Soni R 2016 An improved functional link artificial neural networks with intuitionistic fuzzy clustering for software cost estimation. International Journal of System Assurance Engineering and Management 7: 50-61

[2] Mittas N, Papatheocharous E, Angelis L and Andreou A S 2015 Integrating non-parametric models with linear 
components for producing software cost estimations. Journal of Systems and Software 99: 120-134

[3] Kapoor D and Gupta R K 2016 Software cost estimation techniques - a review of literature. International Journal of Research and Development in Applied Science and Engineering 9

[4] Hodgkinson A C and Garratt P W 1999 A neuro fuzzy cost estimator. In: Proceedings of the Third International Conference on Software Engineering and Applications-SAE, pp. 401-406

[5] Borade J G 2013 Software project effort and cost estimation techniques. International Journal of Advanced Research in Computer Science and Software Engineering 3: 730-739

[6] Rao K S and Reddy L S S 2013 Software cost estimation in multilayer feed forward network using random holdback method. International Journal of Advanced Research in Computer Science and Software Engineering 3: 1309-1328

[7] Mukherjee S, Bhattacharya B and Mandal S 2013 A survey on metrics, models \& tools of software cost estimation. International Journal of Advanced Research in Computer Engineering \& Technology 2: 2620-2625

[8] Ramasubbu N and Balan R K 2012 Overcoming the challenges in cost estimation for distributed software projects. In: Proceedings of the 34th International Conference on Software Engineering (ICSE), pp. 91-101

[9] Srinivasan K and Fisher D 1995 Machine learning approaches to estimating software development effort. IEEE Transactions on Software Engineering 21: 126-137

[10] Shivhare J and Rath S K 2014 Software effort estimation using machine learning techniques. In: Proceedings of the 7th India Software Engineering Conference, pp. 19-21

[11] Araújo R A, Soares S and Oliveira A L I 2012 Hybrid morphological methodology for software development cost estimation. Expert Systems with Applications 39: 6129-6139

[12] Araújo R A, Oliveira A L I, Soares S and Meira S 2012 An evolutionary morphological approach for software development cost estimation. Neural Networks 32: 285-291

[13] Maleki I, Ghaffari A and Masdari M 2014 A new approach for software cost estimation with hybrid genetic algorithm and ant colony optimization. International Journal of Innovation and Applied Studies 5: 72-81

[14] Sadiq M and Shahid M 2013 A systematic approach for the estimation of software risk and cost using esrcTool. CSI Transactions on ICT 1: 243-252

[15] Mittas N and Angelis L 2013 Ranking and clustering software cost estimation models through a multiple comparisons algorithm. IEEE Transactions on Software Engineering 39: 537-551

[16] Bishnu P S and Bhattacherjee V 2016 Software cost estimation based on modified K-modes clustering algorithm. Natural Computing 15: 415-422

[17] Roli F 2009 Multiple classifier systems. In: Encyclopedia of Biometrics, pp. 981-986

[18] Sharma R 2013 Survey: Non algorithmic models for estimating software effort. European International Journal of Science and Technology 2: 164-169

[19] Waghmode S and Kolhe K 2014 A novel way of cost estimation in software project development based on clustering techniques. International Journal of Innovative Research in Computer and Communication Engineering 2: 3892-3899
[20] Mittas N and Angelis L 2008 Comparing cost prediction models by resampling techniques. Journal of Systems and Software 81: 616-632

[21] Stensrud E and Myrtveit I 1998 Human performance estimating with analogy and regression models: an empirical validation. In: Proceedings of the Fifth IEEE International Symposium on Software Metrics, pp. 205-213

[22] Mogale D G, Lahoti G, Jha S B, Shukla M, Kamath N and Tiwari M K 2018 Dual market facility network design under bounded rationality. In: Proceedings of Algorithms 2018, 11: 54

[23] Kitchenham B and Mendes E 2009 Why comparative effort prediction studies may be invalid. In: Proceedings of the Fifth ACM International Conference on Predictor Models in Software Engineering, pp. 1-5

[24] Mogale D G, Kumar M, Kumar S K and Tiwari M K 2018 Grain silo location-allocation problem with dwell time for optimization of food grain supply chain network. Transportation Research Part E: Logistics and Transportation Review 111: 40-69

[25] Maiyar L M and Thakkar J J 2018 Modelling and analysis of inter-modal food grain transportation under hub disruption towards sustainability. International Journal of Production Economics. https://doi.org/10.1016/j.ijpe.2018.07.021

[26] Mogale D G, Kumar S K and Tiwari M K 2018 An MINLP model to support the movement and storage decisions of the Indian food grain supply chain. Control Engineering Practice 70: 98-113

[27] Elbeltagi E, Hegazy T and Grierson D 2005 Comparison among five evolutionary-based optimization algorithms. Advanced Engineering Informatics 19: 43-53

[28] Dizaji Z A and Gharehchopogh F S 2015 A hybrid of ant colony optimization and chaos optimization algorithms approach for software cost estimation. Indian Journal of Science and Technology 8: 128-133

[29] Gharehchopogh F S, Maleki I and Talebi A 2015 Using hybrid model of artificial bee colony and genetic algorithms in software cost estimation. In: Proceedings of the 9th International Conference on Application of Information and Communication Technologies (AICT), Rostov on Don, Russia

[30] Schneider A, Hommel G and Blettner M 2010 Linear regression analysis. Evaluation of Scientific Publications, Part 14

[31] Smola A and Scholkopf B 2000 Sparse greedy matrix approximation for machine learning. In: Proceedings of the Seventeenth International Conference on Machine Learning. Morgan Kaufmann, San Francisco, CA, USA

[32] Askarzadeh A 2016 A novel metaheuristic method for solving constrained engineering optimization problems: crow search algorithm. Computers and Structures 169: 1-12

[33] Mirjalili S and Lewis A 2016 The Whale Optimization Algorithm. Advances in Engineering Software 95: 51-67

[34] Promise software engineering repository 2017 "http://promise. site.uottawa.ca/SERepository/datasets-page.htmlc”, accessed in October.

[35] Gharehchopogh F S, Ebrahimi L, Maleki I and Gourabi S J 2014 A novel PSO based approach with hybrid of fuzzy $\mathrm{C}$-means and learning automata in software cost estimation. Indian Journal of Science and Technology 7: 795-803 
[36] Gharehchopogh F S, Rezaii R and Arasteh B 2015 A new approach by using Tabu search and genetic algorithms in software cost estimation. In: Proceedings of the 9th International Conference on Application of Information and Communication Technologies (AICT), Rostov on Don, Russia
[37] Maiyar L M and Thakkar J J 2017 A combined tactical and operational deterministic food grain transportation model: particle swarm based optimization approach. Computers \& Industrial Engineering 110: 30-42 